\title{
Impact of Pressure Injury Prevention Protocol in Home Care Services on the Prevalence of Pressure Injuries in the Dubai Community
}

\author{
Sajitha Prasad ${ }^{a}$ Nazneen Hussain ${ }^{b}$ Sangeeta Sharma ${ }^{c}$ Somy Chandy ${ }^{d}$ \\ Jessy Kurien ${ }^{d}$
}

a Family Medicine Department, PHCS, Al Mizhar Health Center, Dubai Health Authority, Dubai, UAE; ${ }^{\mathrm{b}}$ Family Medicine Department, PHCS, Al Khwaneej Health Center, Dubai Health Authority, Dubai, UAE; ' $F$ amily Medicine Department, PHCS, Al Lusaily Health Centre, Dubai Health Authority, Dubai, UAE; ${ }^{d}$ Nursing Department, PHCS, Nad al Hamar Health Centre, Dubai Health Authority, Dubai, UAE

\section{Keywords}

Guideline · Pressure injuries · Prevalence · Home care · United Arab Emirates

\section{Abstract \\ Background: Pressure injuries (PIs) in the community have emerged as a health care burden in the past few years, lead- ing to high rates of morbidity and mortality among the el- derly population. There is evidence that simple risk assess- ment tools and protocols have reduced the prevalence of Pls considerably by shifting the focus to timely prevention and adequate management. The prevalence of Pls is high in home care setting and utilizes a major share of the organiza- tional resources for its treatment and prevention. Aim: This study aims to assess the impact of the newly developed PI prevention protocol for home care patients in Dubai. The ob- jectives are to evaluate effective implementation of the pro- posed protocol and its impact on the prevalence of Pls in the community to identify the gaps for improvement in the fu- ture. Methods: This retrospective observational was con- ducted in 13 primary health care centers in Dubai, UAE. Data were collected from 249 patients' records at an average age}

of $75.5 \pm 14.5$ years old with compromised mobility (bedbound/chairbound) from January to July 2019. The PI prevalence was assessed before and after 6 months of implementing the PI prevention protocol and comparison was done using a standardized skin assessment scale (Braden Scale). Internationally validated tools from the Agency for Healthcare Research and Quality and National Institute for Health and Care Excellence were used to ensure the reliable use of the Braden Scale and PI protocol compliance. The prevalence was calculated from the existing key performance indicators in the home care office and considering the significant improvement at $p$ value of $<0.05$. Results: The findings in the first quarter revealed a significant drop in both prevalence $(9.0 \%)$ and incidence rate $(6.0 \%)$ to approximately $2.0 \%$. Overall PIs prevalence declined significantly after implementing the protocol $(p<0.0001)$ among both genders. Also, a significant improvement was detected in the use of Braden Scale and multidisciplinary care plan $(p<0.0001)$. Conclusion: This study indicates that standardization of care delivery reduces the increased risk and incidence of Pls with a potentially positive outcome on PI prevalence.

(c) 2020 The Author(s).

Published by S. Karger AG, Basel karger@karger.com www.karger.com/dmj

Karger $\stackrel{\text { ' }}{5}$

BOPEN ACCESS
(C) 2020 The Author(s)

Published by S. Karger AG, Basel

This article is licensed under the Creative Commons AttributionNonCommercial-NoDerivatives 4.0 International License (CC BYNC-ND) (http://www.karger.com/Services/OpenAccessLicense) Usage and distribution for commercial purposes as well as any distribution of modified material requires written permission.
Sajitha Prasad

Family Medicine Department, PHCS, Al Mizhar Health Center Senior Specialist Registrar, Dubai Health Authority PO Box-4545 Dubai (UAE) sprasad@dha.gov.ae 


\section{Introduction}

Pressure Injuries (PIs) in the community have emerged as a health care burden in the past few years and the morbidity and mortality among the elderly population have been on the rise due to it [1]. There is an evidence that simple risk assessment tools and protocols have reduced the prevalence of PIs considerably by shifting the focus to timely prevention and adequate management [2]. The prevalence of PIs is high in home care setting and utilizes a major share of the organizational resources for its treatment and prevention. Extended pressure on body parts affects circulation leading to weak tissue perfusion, ischemia, and necrosis along with chronic medical conditions which are major determinants of PI [3]. Considering the adverse effects on healthcare costs and patient morbidity Agency for Healthcare Research and Quality (AHRQ), Stage III and IV PI have been listed as a never-ending phenomenon [4].

A unique customized PI prevention protocol specifically for home care services was developed and introduced to standardize the home care delivery process within the Primary Healthcare Services Department in Dubai. This research is aimed at studying the effectiveness of this protocol used in-home care and to identify the gaps for improvements. The protocol acts as a directive for home care staff to implement preventive and systematized patient care measures through internationally validated best practices to avoid any threatening event [5].

The primary source of adverse outcomes in healthcare is flawed processes rather than human ones and the quintessential quality dimensions, namely patient-centeredness, timeliness, effectiveness, efficiency, and safety, play an integral role in health care [1]. Unlike restricted organizational environments, the nature of home care is very complex where the frequency of adverse outcomes is as high as 3.5-15.1 percent [2]. It enforces the need for strict guidelines and procedures for medical treatment to ensure optimal patient safety to reduce adverse outcomes such as PIs [3]. Home care workers evaluation reported awareness gaps in PI evaluation and prevention approaches for approximately 80 percent of home care nurses within the organization. There was no evidence that the home care team provides the caregivers with any education on the topic. This lack of caregiver preparation has been described as a root cause of PI, occurring worldwide [6].

The homecare team carries out comprehensive risk assessment using standardized tools like Braden Scale for PIs risk. Lack of continuity in evaluating risk factors, im- plementing preventive measures, and subsequent reassessments were found to be a cause for rising prevalence of PIs in home care. The new protocol on prevention of PIs, therefore, was implemented to shift the focus on the effective use of Braden Scale assessment along with the implementation of standardized preventive approach recommendations [7].

\section{Methods}

This study was conducted in 13 primary health care centers in Dubai, UAE. Registered home care patients with compromised mobility (bedbound/chairbound) and comorbid chronic debilitating illness like diabetes, hypertension, cerebrovascular accidents of $75.5 \pm 14.5$ years of age were included in the study. Data collection was initiated after the Dubai Scientific Research Ethics Committee approved the project (Ref: DSREC-09/2019_11). The researcher and collaborator were previously trained to classify PIs and evaluate risk using the Braden Scale and do appropriate data collection.

Currently, 1,200 patients are registered for home care under the 13 operating units. The research team decided to select a sample size using the National Health Service sample size calculator to be 250 [8]. For the total population size of 1,200 , a high confidence level of $95.0 \%$ with an accuracy of $\pm 5.0 \%$ and an expected prevalence of $50.0 \%$ were set as the effectiveness of the control environment in-home care which is low. These values were entered into the online National Health Service Bristol statistical calculator to quickly calculate the study sample size. The expected response rate in this study is $\sim 90.0-100.0 \%$.

The study was conducted in 2 phases. In the first phase, data were collected for patients at risk for pressure injuries from January to September 2018 using the Braden Scale. For the second phase, data were collected from October 2018 to July 2019 after implementation of PI prevention protocol using the same risk assessment tool. Two steps were followed in the study, namely, to estimate the compliance for the PI prevention protocol and impact of the protocol on the PI prevalence in home care service comparing pre- and post-protocol implementation.

The home care nurses recorded the prevalence and incidence data on a PI data collection sheet prepared by the Skin Integrity Team (SIT) on a daily basis and then compiled on a monthly basis in the data sheet. From the compiled sheet, the monthly prevalence and incidence rates were calculated by dividing the number of patients identified with new and existing PIs over the total number of home care patients at risk of PI. Home care office nurse coordinator then plotted the results in the monthly indicators report and sent it to the unit champions.

Data collected from home care registers and records documenting the risk of PIs using the Braden Scale with a predictive power $(r=0.97, p<0.0001)$ [9]. The Braden Scale for Predicting Pressure Sore Risk 10 measures cumulative risk for PI development based on 7 risk factors measured on 6 subscales (Sensory Perception, Activity, Mobility, Moisture, Nutrition, and Friction/ Shear) and is based on the conceptual schema developed by Braden and Bergstrom. Twenty-seven subscale scores range from 1 to 4 with the exception of the Friction/Shear subscale, which ranges from 1 to 3 . Each subscale score is clearly defined by narrative de- 


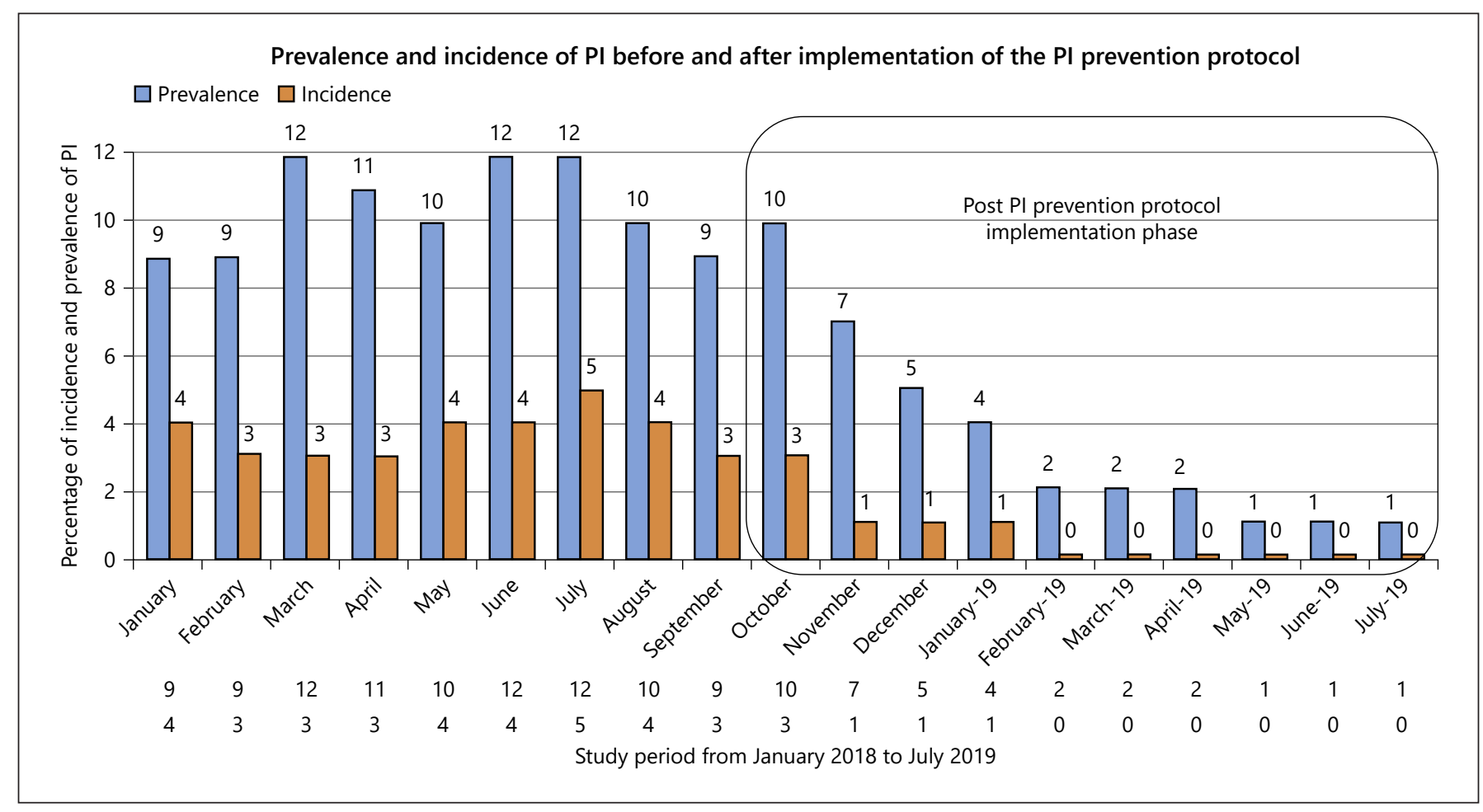

Fig. 1. Prevalence and incidence of PI before and after prevention protocol implementation. PI, pressure injury.

scriptors that assist the clinician to accurately "match" the patient's status to the correct subscale level. Pressure ulcer risk is based on a summated score of 6 to 23, with lower scores indicating greater risk. Currently, a cutoff score of 18 has been found to demonstrate the best balance between sensitivity and specificity; thus, clinically this score represents risk for PI development [9].

The prevalence of PI risk was documented in the validated data collection tools. Internationally validated tools from the AHRQ that offer practical, research-based tools and other resources to help a variety of healthcare organizations, providers, and others make care safer in all health care settings. The staff PI attitude and knowledge survey used to assess the reliable use of Braden Scale and PI protocol compliance assessment tool were adapted for use. The survey using the AHRQ staff attitudes, perception, and knowledge survey tools was conducted after 1 month of the protocol implementation to identify the barriers in carrying out the risk assessment and prevention strategies as per the protocol. The protocol compliance assessment tool used was adapted from the National Institute for Health and Care Excellence as the tool was standardized and validated to produce credible data collection [10].

The clinical nurse specialists in the SIT serve as expert assessors who compare the score of the field nurses and their own. Both the nurse and the SIT nurse assess the same patient independently on the same visit and record it on the inter-rater audit sheet by direct observation, patients' skin inspection, and by interviewing the caregivers. Following this, the data were entered into the assessment tool. The number of "yes" and "non-applicable" sections was calculated as $10 \%$ each. The total score was then calculated to determine the compliance percentage.

PI Prevention Protocol in Home Care Services and the Prevalence of PIs
Categorical results were obtained on the prevalence of the PI rate based on age, gender, protocol elements before/after implementing it and analyzing the compliance assessment statistics. The Statistical Package of Social Sciences software, Version 19.0 for Windows, was used to incorporate the existing key performance indicators and compare the outcomes after implementing the protocol compared to implementing before in the home care office, Dubai Health Authority using McNemar's test. The statistical significance was considered at $p$ value of $<0.05$.

\section{Results}

\section{PI Prevalence}

A total number of 249 patients' records were included in this study according to the inclusion criteria (response rate $\sim 99.6 \%$ ). From January to September 2018, the range of PI prevalence rate was from 9.0 to $12.0 \%$ and the incidence rate was 4.0-6.0\%. However, from October 2018 to July 2019 there was a rapid decline in both prevalence and incidence rate to approximately $2.0 \%$ (as shown in Fig. 1). The steady drop can be attributed to the shift in practice following the implementation of the new PI prevention Protocol and continuous monitoring of high-risk patients dictated by it. Even though the protocol was imple- 


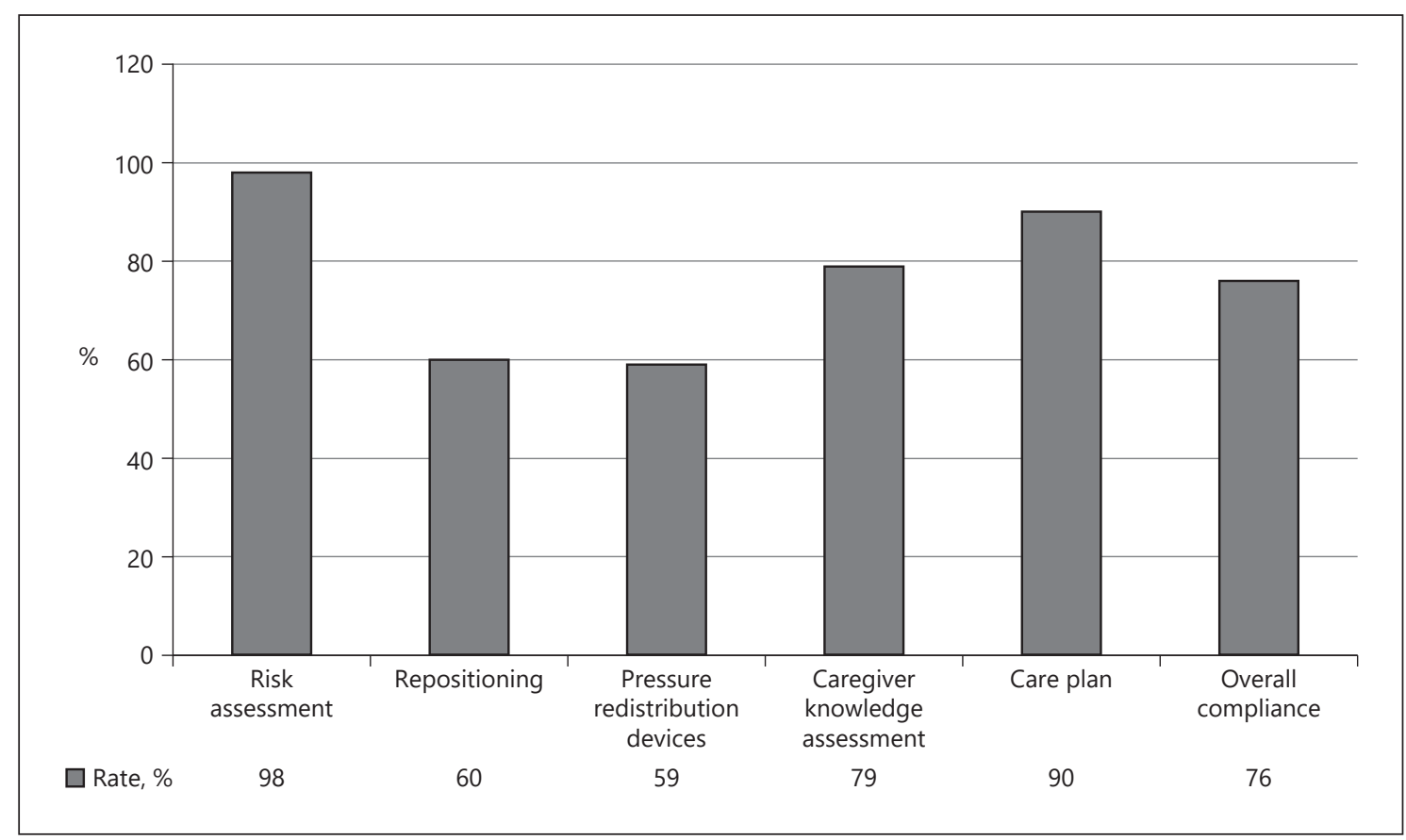

Fig. 2. Protocol compliance assessment.

mented in October 2018, the changes in trend were noted only by January 2019 (after 3 months). This window period was the time taken by the home care nurses to familiarize and incorporate the protocol steps into their routine home care practice.

\section{Protocol Compliance}

The overall protocol compliance assessment done in the home care department after the protocol implementation showed $76.7 \%$ overall compliance (Fig. 2). The results revealed that the issues were related to use of pressure distribution devices, regular patient repositioning, and caregiver knowledge assessment. However, the major underpinning factors are the short time period for protocol implementation, high number of home care nurses and patient turnover. The most significant positive finding noted was the nearly $100.0 \%$ compliance to use of Braden Scale to quantify the PI risk for all home care patients, consistent instructions for repositioning, and an appropriate care plan addressing the identified risk groups.

Table 1 represents a summary of the entire data set including categorical values along with the missing values of each variable. It indicates that out of 249 people, $76.7 \%$ $(n=191)$ had ulcer before the protocol and after implementation of protocol, this percentage declined signifi- cantly $(p<0.0001)$ to almost $23.3 \%(n=58)$. There is clear indication of improvement of $53.4 \%$ among both genders. It was found that out of 249 people, 157 females (79.6\%) had ulcer before the protocol and almost quarter of this number still had ulcer after the protocol implementation $(n=40,20.3 \%)$ with a statistical significance of $p<0.0001$. Similarly, 34 males $(65.4 \%)$ had ulcer and the prevalence of ulcer declined significantly $(p<0.0001)$ to almost half of this number $(n=18,34.6 \%)$. Additionally, there was a significant improvement in Braden Scale, caregivers' knowledge as well as the multidisciplinary care plan $(p<0.0001)$ as shown in Table 1 , which reflects the effectiveness of the newly implemented protocol.

\section{Discussion/Conclusion}

Implementation of best practices is a challenge for most institutions and even more challenging in home care which was successfully attempted by the DHA primary care by grouping the PI prevention recommendations into a customized home care protocol. This package or intervention influenced the performance of nurses with a noted increase in the adoption of evidence-based recommendations on PI prevention in the community [11]. 
Table 1. Impact of PI prevention protocol on PI prevalence and prevention strategies

\begin{tabular}{|c|c|c|c|c|c|c|c|c|c|c|}
\hline & \multicolumn{4}{|c|}{ Cases } & \multicolumn{4}{|c|}{ Prevalence of PI } & \multirow[t]{2}{*}{$p$ value } & \multirow{2}{*}{$\begin{array}{l}\text { Positive } \\
\text { impact, } \\
\%\end{array}$} \\
\hline & $N$ & $\%$ & $N$ & $\%$ & $N$ & $\%$ & $N$ & $\%$ & & \\
\hline Overall PI prevalence & 249 & 100.0 & 0 & 0.0 & 191 & 76.7 & 58 & 23.3 & $<0.0001$ & $>53.4$ \\
\hline PI prevalence in females & 197 & 79.1 & 0 & 0.0 & 157 & 79.6 & 40 & 20.3 & $<0.0001$ & $>59.3$ \\
\hline PI prevalence in males & 52 & 20.5 & 0 & 0.0 & 34 & 65.4 & 18 & 34.6 & $<0.0001$ & $>30.8$ \\
\hline PIs risk using Braden Scale & 249 & 100.0 & 0 & 0.0 & 58 & 23.3 & 191 & 96.7 & $<0.0001$ & $>73.4$ \\
\hline Caregiver knowledge test & 222 & 89.2 & 27 & 10.8 & 7 & 3.2 & 215 & 96.8 & $<0.0001$ & $>93.6$ \\
\hline Repositioning every $6 / 2 \mathrm{~h}$ & 139 & 55.8 & 110 & 44.2 & 61 & 43.8 & 78 & 56.1 & $<0.0001$ & $>12.3$ \\
\hline Use of patient turning clock & 175 & 70.3 & 74 & 29.7 & 33 & 18.9 & 142 & 81.1 & $<0.0001$ & $>62.2$ \\
\hline Use of alternate pressure mattress & 184 & 73.9 & 65 & 26.1 & 45 & 24.5 & 139 & 75.5 & $<0.0001$ & $>51.0$ \\
\hline Use of heel cap & 163 & 65.5 & 86 & 34.5 & 41 & 25.2 & 122 & 74.8 & 0.0001 & $>49.6$ \\
\hline
\end{tabular}

PI, pressure injury. $p$ values $<0.05$ (in bold typeface) indicate that the result that was observed is quite unlikely to occur when the null hypothesis is true; hence, this result suggests strongly that the null hypothesis is false.

The major hurdles in compliance appear to be related to knowledge deficit in relation to PI prevention along with the erratic supply of prevention materials and workforce for continuous implementation in the community as noted worldwide [12].

The nurses in project focused on 2 critical things for risk assessment namely the presence of risk factors for PI like hypoperfusion states, diabetes, etc. and the presence of PI before devising a care plan [13]. Care plan majorly focused on utilizing the risk assessment results to guide the treatment, ensure patient safety, and as an educational tool for the patient family and caregivers', which was provided for more than $90 \%$ (Fig. 2) of the patients. Although the protocol reflected best practice evidence, its success is founded on nurses and patient caregivers' participation. This active engagement and partnership are determined by the efficacy of training along with the behavior, attitudes, perceptions, and competency levels of the participating individuals [14].

Increased compliance with evidence-based PI prevention strategies is deemed as an achievement in dynamic home care environment. The customized protocol, therefore, proved to be a driver for sustainable evidence-based practice change, which demonstrates the need for community-focused PI risk assessment tools and education of caregivers along with the home care nurses. However, an adaptation of PI protocol in-home health care setting is more complex than that in hospitals and nursing homes, which requires significant skills in communication and collaboration [15].

Additional research on the most effective PI practices and wound care products that are cost-efficient and costeffective for the clients would be beneficial in achieving improved client care outcomes. To overcome the limitations in protocol implementation, liaison with other primary care sections may be mandated. Inclusion of caregiver training and in-service training for nurses to address, reinforce, and update PI prevention knowledge may prove beneficial. An interdisciplinary team of home care nurses, coordinators, and case managers can ensure the protocol sustenance. Future research and audits to guarantee sustained evidence-based practice changes are necessary. Furthermore, "How strongly the organization's values and message correlate with better nursing care in regards to pressure ulcer prevention" is an important and interesting question worth exploration.

\section{Acknowledgements}

The authors thank PHCS SIT for their support.

\section{Statement of Ethics}

Dubai Scientific Research Ethics Committee approved the project (Ref: DSREC-09/2019_11). 


\section{Conflict of Interest Statement}

Authors have no conflict of interest.

\section{Funding Sources}

No funding used.

\section{References}

1 Berwick DM. A user's manual for the IOM's 'quality chasm' report. Health Aff. 2002 May; 21(3):80-90.

2 Masotti P, McColl MA, Green M. Adverse events experienced by homecare patients: a scoping review of the literature. Int J Qual Health Care. 2010 Apr 1;22(2):115-25.

3 Lang A, Edwards N, Fleiszer A. Safety in home care: a broadened perspective of patient safety. Int J Qual Health Care. 2007;20(2):130-5.

4 Brindle CT, Wegelin JA. Prophylactic dressing application to reduce pressure ulcer formation in cardiac surgery patients. J Wound Ostomy Continence Nurs. 2012 Mar 1;39(2): $133-42$.

5 Indicators AQ. Agency for healthcare research and quality, 2015.

6 Chou R, Dana T, Bougatsos C, Blazina I, Starmer AJ, Reitel K, et al. Pressure ulcer risk assessment and prevention: a systematic comparative effectiveness review. Ann Intern Med. 2013 Jul 2;159(1):28-38.

\section{Author Contributions}

S.P.: design of the study and result interpretation; N.H.: methodology and discussion; S.S.: approval and conclusion; S.C. and J.K.: data collection and interpretation.
7 KottnerJ HR, Dassen T. An interrater reliability study of the assessment of pressure ulcer risk using the Braden scale and the classification of pressure ulcers in a home care setting. Int J Nurs Stud. 2009 Oct 1;46(10):1307-12.

8 Bristol UH, NHS. The Sample Size Calculator; 2009.

$9 \mathrm{Na}$ H-J, Yoo S-H, Kwon Y-R, Ahn M-J. The interrater agreement for the assessment of pressure ulcer risk using the braden scale and the classification of pressure ulcers by nurses in a medium-sized hospital. Korean J Adult Nurs. 2020 Feb 1;32(1):35-45.

10 Park KH, Kim JY, Park OK, Park JH, Lee YJ, Hwang $\mathrm{JH}$. Updates of nursing practice guideline for pressure injury. J Korean Clin Nurs Res. 2019;25(1):67-79.

11 Brimelow RE, Wollin JA. The impact of care practices and health demographics on the prevalence of skin tears and pressure injuries in aged care. J Clin Nurs. 2019;27(7-8):151928.
12 Kwong EW, Lee PH, Yeung KM. Study protocol of a cluster randomized controlled trial evaluating the efficacy of a comprehensive pressure ulcer prevention programme for private for-profit nursing homes. BMC Geriatr. 2015;16(1):20.

13 Garcia Fernandez FP, Pancorbo-Hidalgo PL, Agreda JJS. Predictive capacity of risk assessment scales and clinical judgment for pressure ulcers: a meta-analysis. J Wound Ostomy Continence Nurs. 2014;41(1):24-34.

14 Patelarou AE, Patelarou E, Brokalaki H, Dafermos V, Thiel L, Melas CD, et al. Current evidence on the attitudes, knowledge, and perceptions of nurses regarding evidencebased practice implementation in European community settings: a systematic review. J Community Health Nurs. 2013;30(4):230-44.

15 Wong CA, Cummings GG, Ducharme L. The relationship between nursing leadership and patient outcomes: a systematic review update. J Nurs Manag. 2013 Jul;21(5):709-24. 